\title{
Bovine spongiform encephalopathy infection alters endogenous retrovirus expression in distinct brain regions of cynomolgus macaques (Macaca fascicularis)
}

Alex D Greenwood ${ }^{1 \dagger}$, Michelle Vincendeau ${ }^{2,5+}$, Ann-Christin Schmädicke ${ }^{3}$, Judith Montag ${ }^{3,6}$, Wolfgang Seifarth ${ }^{4}$ and Dirk Motzkus ${ }^{3^{*}}$

\begin{abstract}
Background: Prion diseases such as bovine spongiform encephalopathies (BSE) are transmissible neurodegenerative diseases which are presumably caused by an infectious conformational isoform of the cellular prion protein. Previous work has provided evidence that in murine prion disease the endogenous retrovirus (ERV) expression is altered in the brain. To determine if prion-induced changes in ERV expression are a general phenomenon we used a non-human primate model for prion disease.

Results: Cynomolgus macaques (Macaca fasicularis) were infected intracerebrally with BSE-positive brain stem material from cattle and allowed to develop prion disease. Brain tissue from the basis pontis and vermis cerebelli of the six animals and the same regions from four healthy controls were subjected to ERV expression profiling using a retrovirus-specific microarray and quantitative real-time PCR. We could show that Class I gammaretroviruses HERVE4-1, ERV-9, and MacERV-4 increase expression in BSE-infected macaques. In a second approach, we analysed ERV$\mathrm{K}-(\mathrm{HML}-2) \mathrm{RNA}$ and protein expression in extracts from the same cynomolgus macaques. Here we found a significant downregulation of both, the macaque ERV-K-(HML-2) Gag protein and RNA in the frontal/parietal cortex of BSE-infected macaques.

Conclusions: We provide evidence that dysregulation of ERVs in response to BSE-infection can be detected on both, the RNA and the protein level. To our knowledge, this is the first report on the differential expression of ERVderived structural proteins in prion disorders. Our findings suggest that endogenous retroviruses may induce or exacerbate the pathological consequences of prion-associated neurodegeneration.
\end{abstract}

\section{Background}

Prion diseases are fatal transmissible neurodegenerative diseases that include Creutzfeldt-Jakob Disease (CJD) in humans and bovine spongiform encephalopathy (BSE) in cattle [1-4]. A key observation in the development of prion diseases is the accumulation of the abnormal isoform $\left(\mathrm{PrP}^{\mathrm{Sc}}\right)$ of the host encoded prion protein $\left(\operatorname{PrP} \mathrm{P}^{\mathrm{C}}\right)$ in the brain of affected individuals [5,6]. As a result, neuronal loss and astrogliosis lead to the clinical

\footnotetext{
*Correspondence: dmotzkus@dpz.eu

+ Contributed equally

${ }^{3}$ German Primate Center, Leibniz-Institute for Primate Research, Unit of Infection Models, D-37077 Göttingen, Germany

Full list of author information is available at the end of the article
}

symptoms associated with prion diseases such as ataxia and dementia. The mechanism by which $\operatorname{PrP}^{\mathrm{C}}$ is converted to $\mathrm{PrP}^{\mathrm{Sc}}$ is not well understood. As a result, several researchers have hypothesized that host-derived nucleic acids or other non-PrP molecules contribute to the propagation of prions [7-10]. In search of these unknown factors, multiple studies in mice and humans have examined host gene expression response to prioninfection revealing multiple biochemical pathways that are disturbed. These altered pathways may contribute to prion disease pathology [11-22] though most studies have yielded conflicting results among tissue types, methods, and cell lines used [23].

\section{C) Biomed Central}


One group of sequences with altered expression as a consequence of prion infection in both, cell lines and tissue samples from humans and mice, are endogenous retroviruses (ERVs) [24-28]. ERVs are the remnants of germline infection by relatives of exogenous retroviruses such as murine leukemia virus (MLV) and mouse mammary tumor viruses (MMTV) [29]. Endogenization is a frequent event as the genomes of all mammals studied are composed of $8-11 \%$ retrovirus-like sequences. They are not only passive components of the genome or "Junk DNA", but can also play important functional roles. For example the ERV-derived proteins syncytin-1 and -2 are essential for trophoblast differentiation in placental development [30-33]. The long terminal repeats (LTRs), which control retroviral gene expression can also change host RNA levels [29]. Additionally, the ERV-expression pattern has been shown to be altered in a murine cell culture model for prion infection [25] and in the cerebrospinal fluid (CSF) of sporadic CJD patients [28]. The PrP and ERV expression in some immunestimulated mouse tissues also appear to be linked [34]. Infections of mice with ecotropic neurovirulent retroviruses can also be associated with prion-like disorders. The MLV strains Cas-Br-E, Friend MLV Fr98 and the ts-1 mutant of MoMLV cause neuropathology that is similar to prion-induced spongiosis [35-37]. Interestingly, the prion gene is totally dispensable in the pathogenesis and tropism of Cas-Br-E [36]. This lead to the conclusion that retroviral-induced neurodegeneration may act in a common pathway but downstream of prion infection.

To date, the expression of endogenous retroviruses in human CJD has only been analysed in patients' CSF samples [28]. ERV profiling in brain tissue from human CJD has not been performed. A systematic analysis in a controlled experimental non-human primate model for prion disease provides the unique opportunity for examining the expression of ERVs in response to infectious prions in the primate brain. Cynomolgus macaques are an excellent model for human acquired prion disease. TSEs can be transmitted to Macaca fascicularis leading to disease that is comparable to humans, including preclinical incubation time, clinical symptoms and pathophysiology [38]. For ERV expression analysis, a microarray system for the detection of human endogenous retroviral expression has been successfully applied to genomic DNA and RNA derived from rhesus macaque tissues (Macaca mulatta) [39] or brain tissue from barbary macaques (Macaca sylvanus) [40].

The aim of our study was to assess the putative dysregulation of endogenous retroviruses on the transcriptional and protein level linked to prion pathology. For this reason we analyzed three defined brain regions, vermis cerebelli, basis pontis and the frontal/parietal cortex.
The samples from the infected macaques were compared to samples from the same brain regions of non-infected control animals. Changes in gene expression were examined using a retrovirus-specific microarray [40] and quantitative real-time PCR (QPCR). Western blot analysis with an HERV-K-(HML-2) Gag specific antiserum was used to investigate changes at the protein level.

\section{Methods}

\section{Tissues and RNA extraction}

Cynomolgus macaques (Macaca fascicularis) were purchased from the Centre de Recherche en Primatologie, Mauritius and maintained in social groups of six monkeys housed in a microbiological containment level BSB3 facility. Ethics approval for the study was issued by the Lower Saxony Ministry for consumer protection and food safety (509.42502/08/07.98). Animal experimentation was performed in accordance with section 8 of the German Animal Protection Law in compliance with EC Directive 86/609. It should be mentioned that the diet and housing of the used experimental and the control animals were not identical. This may well have contributed to the inter-individual variations among the animals. Six cynomolgus macaques were intracerebrally inoculated with $50 \mathrm{mg}$ bovine BSE-positive brain stem material derived from an EU-standard inoculum. Inoculation was performed by slow injection of $250 \mu \mathrm{l}$ of a $20 \%$ BSE brain homogenate $(\mathrm{w} / \mathrm{v})$ diluted in sucrose into the right hemisphere through the dura mater into the caudo-putamen. The preclinical incubation time was on average 1,100 days. Animals were sacrificed, and the brains were dorso-ventrally sliced and snap-frozen on dry-ice plates. Brain material from age-and sex-matched non-infected cynomolgus macaques was obtained from Covance Laboratory Münster $\mathrm{GmbH}$ and processed in an equivalent manner. Three different brain regions (vermis cerebelli, basis pontis, frontal/parietal cortex) were macroscopically identified on the frozen brain slices, isolated via a bore device and ground to powder under liquid nitrogen (for extraction of RNA) or homogenized (for extraction of protein).

Total RNA (RNA > 200 bases) was isolated from each sample using a mirVANA kit (Ambion). To remove genomic DNA, all RNA samples were treated with $1 \mathrm{U} /$ $\mu$ g RQ1 RNase-free DNase I (Promega). Subsequently, $25 \mathrm{ng}$ of each RNA preparation was tested by PCR with retroviral mixed oligo primers (MOP) as described previously [41] omitting the reverse transcription step. Only RNA preparations negative for amplification products were used for reverse transcription and MOP multiplex PCR. Samples tested positive were treated again with DNase I until the MOP multiplex control PCR were negative. This precaution was taken to ensure 
that all experiments measuring RNA levels were completely DNA-free.

\section{Reverse transcription, PCR and microarray analysis}

Reverse transcription of RNA was performed using $1 \mu \mathrm{g}$ total RNA with Superscript II (Roche Diagnostics) according to the manufacturer's protocol. Reverse transcriptions were followed by an RNase $\mathrm{H}$ digestion step (Promega). Syntheses of hybridization probes, labelling of MOP PCR products, preparation, hybridization and post-processing of retrovirus-specific DNA microarrays were performed as previously described $[41,42]$. The exception was that PCRs were performed with KAPA2G Robust DNA Polymerase (Peqlab Biotechnologie $\mathrm{GmbH}$ ) which was found to improve the microarray results substantially in terms of reproducibility and signal intensity (data not shown).

Hybridized microarrays were scanned using an Affymetrix GMS 418 scanner (laser power setting, 100\%; gain setting, 50\%), and the resulting images (16-bit TIFF files) were subjected to densitometric analysis using ImaGene 4.0 software (BioDiscovery Inc.). To discriminate positive signals from background, a cut-off value of 1,200 relative fluorescence intensity units was used that corresponded to twofold overall background intensity value of the microarray. To account for the influence of RNA quality, HERV signals were normalized to the fluorescence intensity of the hypoxanthine-guanine phosphoribosyltransferase (HPRT) transcript as a housekeeping gene showing the most consistent transcript levels on the microarray. This was confirmed by densitometric analysis which demonstrated that there was no statistically significant difference in HPRT expression difference between infected and uninfected animals (data not shown). Thus, microarray-based relative HERV transcript levels were expressed as a ratio of HERV to HPRT. Microarray data were submitted to Gene Expression Omnibus (GEO: GSE29818).

\section{QPCR}

Primers used for quantitative real time PCR (QPCR) were HERV-E: E4-1 (forward, 5'-GGTGTCACTACTC AATACAC-3'; reverse, 5'-GCAGCCTAGGTCTCTGG3'), MacERV-4 (forward, 5'-ATGTAGCTGCCGCTATAGAG-3'; reverse, 5'-GCTATTTGTAGTCCGGCTGC-3') and ERV-K (HML-2) gag (forward, 5'- TATGATTGGGAGATTCTGGCA-3'; reverse, 5'-GCAGCCCTAT TTCTTCGGAC-3'). The HERV-E, and MacERV-4 primers were previously shown to work on barbary macaques [40]. QPCR was performed with the Roche LightCycler480 System, using LC-480 SybrGreen PCR mix and a standard LightCycler protocol (Roche Diagnostics). Amplifications were performed using a $10 \mathrm{~min}$ denaturation step at $95^{\circ} \mathrm{C}$, followed by 45 cycles of $10 \mathrm{sec}$ at $95^{\circ} \mathrm{C}, 5 \mathrm{sec}$ at $60^{\circ} \mathrm{C}$, and $10 \mathrm{sec}$ at $72^{\circ} \mathrm{C}$. RNA-Polymerase II-transcripts (RPII) were analyzed as a standard, using published primers [43]. For quantification of ERVK (HML-2) a 7500 Real Time PCR System (Applied Biosystem) was used. Reaction was performed after $10 \mathrm{~min}$ denaturation with 45 cycles between $95^{\circ} \mathrm{C}$ for $15 \mathrm{sec}$ and $58^{\circ} \mathrm{C}$ for $45 \mathrm{sec}$ using Power SybrGreen PCR Mastermix (Applied Biosystems) with $2.5 \mathrm{ng}$ RNA equivalent amount and $400 \mathrm{nM}$ of each primer per reaction. The relative expression ratio was calculated as described [44]. Melting curve analysis and gel electrophoresis of amplification products was performed to verify that artefact products or primer dimers were not responsible for the signals obtained (see Additional file 1: Figure S3).

\section{Western blot analysis}

Frontal/parietal cortex samples were homogenized in sample buffer $(62.5 \mathrm{mM}$ Tris/ $\mathrm{HCl}, \mathrm{pH} 6.8,62.5 \mathrm{mM}$ Imidazol, $\mathrm{pH}$ 6.8, 10\% SDS, 0.01\% bromphenolblue, $12.5 \%$ glycerol, $2.5 \%$ beta-mercaptoethanol) at $20 \%(\mathrm{w} / \mathrm{v})$ and stored at $-80^{\circ} \mathrm{C}$ prior to use. Extracts were spun for $2 \mathrm{~min}$ at 7,000 $\times \mathrm{g}$ and the supernatant was diluted 1:1 in LDS sample buffer (Invitrogen). 10\% Glycerol (v/v) was added and samples were incubated for $5 \mathrm{~min}$ at $95^{\circ}$ $\mathrm{C}$ and cooled on ice. For immunoblotting, $10 \mu \mathrm{l}$ of supernatant was separated on a $12 \%$ Bis-Tris polyacrylamide gel in MOPS-SDS running buffer (NuPAGE, Invitrogen) together with a molecular weight marker (Spectra Multicolor Broad Range Protein Ladder, Fermentas) for $150 \mathrm{~min}$ at $30 \mathrm{~mA}$. The gel was blotted onto a PVDF membrane (Immobilon-P transfer membrane $0.45 \mu \mathrm{m}$, Millipore) in transfer buffer (NuPAGE, Invitrogen) for $70 \mathrm{~min}$ at $135 \mathrm{~mA}$. Prion infectivity of the blot was inactivated by incubation in $4 \mathrm{M}$ Guanidinium-Thiocyanate (Roth) for $30 \mathrm{~min}$ at room temperature with slow agitation. The PVDF was washed in TBS and unspecific binding was blocked with $0.2 \%$ Casein (I-Block, Applied Biosystems) for $30 \mathrm{~min}$ at room temperature. As primary antibody, a polyclonal antiserum (K2548) that was raised against full length HERV-K(HML-2) Gag [45] was used at a dilution of $10^{-2}$. This antiserum was generated as described for serum 6897 [45]. A pre-immune serum derived from the same animal was used as a negative control. As a loading control, a $\beta$-actin antibody (mAbcam 8224) was used at a dilution of $10^{-3}$. Antibody incubation was performed overnight at $4{ }^{\circ} \mathrm{C}$ in $\mathrm{TBS} / 0.02 \%$ Casein. Detection was carried out using anti-rabbit IgG-AP (A9919, Sigma-Aldrich) at a dilution of $10^{-5}$ in TBS/ $0.02 \%$ Casein for $1 \mathrm{~h}$ at room temperature with gentle agitation. Bands were visualized with NBT/BCIP substrate (Moss Inc.) for $10 \mathrm{~min}$. The reaction was stopped by addition of $\mathrm{dd}_{2} \mathrm{O}$ and scanned. Densitometric analysis was performed with the program Image J $1.37 \mathrm{v}$. 


\section{Statistics}

Data analysis was performed using GraphPad Prism 5.0. Significance was calculated using unpaired student t-test. The corresponding results are indicated in the figures (***, p $<0.001 ; * *, p<0.01 ; *, p<0.05$ ).

\section{Results}

ERV expression profiling in two distinct brain regions of cynomolgus macaques

We investigated whether the expression of endogenous retroviruses was influenced by the infection with prions. RNA was prepared from brain tissue of BSE-infected and non-infected macaques at an advanced stage of prion disease and subjected to reverse transcription with random hexamer primers. Prior to cDNA syntheses, the DNA-free status of each RNA sample was confirmed to prevent interference from DNA copies of ERVs with the microarray probes. It should be noted that the microarray detects HERV groups and not individual HERVs. Thus, signals arising from a HERV group do not necessarily identify specific elements responsible for the signal.

Initially we investigated the general expression, or core profile, of ERVs derived from four non-infected cynomolgus macaques in two distinct brain regions, the basis pontis and the vermis cerebelli (Figure 1, for a full profile also see Additional file 1). The vast majority of the human derived oligonucleotides on the microarray cross-hybridized with macaque ERVs as has been observed for Old World Monkeys in general [39]. ERV profiling revealed high expression of class I gammaretroviruses HERV-E (E4-1, Seq32), HERV-F (HERV-Fb), HERV-W, ERV9 (ERV9, Seq59), class II betaretroviruses HERV-K-(HML-3: Seq26, Seq34, HML-3), HERV-K(HML-10): (HERV-KC4) and macaque specific ERVs (MacERV-1, -2, -3, and -5). Among the animals we detected inter-individual differences in the respective

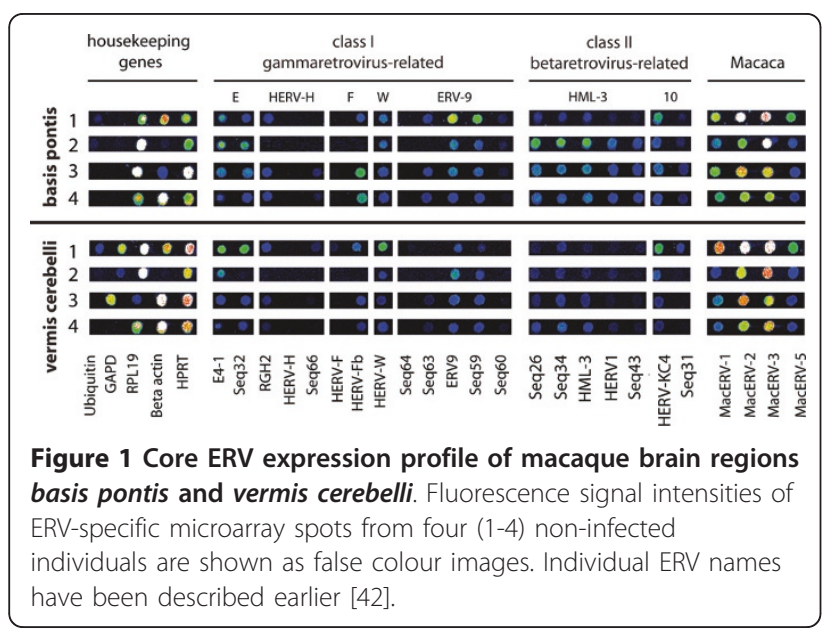

expression pattern. Such differences have also been reported for human studies [46], likely as a result from the non-inbred genetic background of the tested individuals. However, the respective profiles of basis pontis and vermis cerebelli regions were comparable among the macaques.

Comparison of the derived ERV-expression profile in macaques to that of healthy human brain samples [46] revealed a partial overlap of the expressed ERVs including HERV-E4-1, ERV9, Seq59 and HERV-KC4. In contrast, HML-3 elements Seq26, Seq34 and Seq43, that were not or only barely detectable in human prefrontal cortex [46] were highly expressed in basis pontis of macaques. Our results are consistent with previous studies on both DNA and RNA from other non-human primate species that showed both generally conserved presence and expression patterns for several ERVs and additional species specific patterns $[39,40]$.

\section{Differential expression of ERVs in BSE-infected macaques}

Next we used the retrovirus-specific microarray to compare the ERV-expression patterns of BSE-infected with that of non-infected macaques. Quantitative comparison in two different brain regions revealed statistically significant differences between BSE-infected and non-infected macaques (Figure 2). In the infected animals pathogenic prion protein $\left(\mathrm{PrP}^{\mathrm{Sc}}\right)$ was detected in the vermis cerebelli and basis pontis as confirmed by Western blot analysis (data not shown). A detailed description of the distribution of macaque-adapted BSE will be reported elsewhere. Increased expression in BSE-affected macaques was detected for class I elements ERV-E4-1, the macaque specific MacERV-4 and the ERV-9 elements Seq60, ERV9, Seq59. It should be noted that, although statistically significant, upregulation of MacERV-4 seemed to be mainly driven by outliers, as compared to the robust increase of expression in E4-1 and ERV-9 indicating that for some affected ERVs, there is interidividual variability in response to prions. However, upregulation of these endogenous retroviruses was detected in both, basis pontis and vermis cerebellis.

Class II element ERV-K-(HML-3) Seq43 was statistically significant by microarray but also driven by outliers (Additional file 1: Figure S2). None of the Class III HERV-L related elements showed a consistent change in expression. This was not surprising as they are related to foamy viruses which do not cause any known pathological effects as exogenous viruses and are widespread in the genome of mammals indicating they have been endogenized for tens of millions of years. No other changes in ERV expression reached statistical significance for either brain region. 


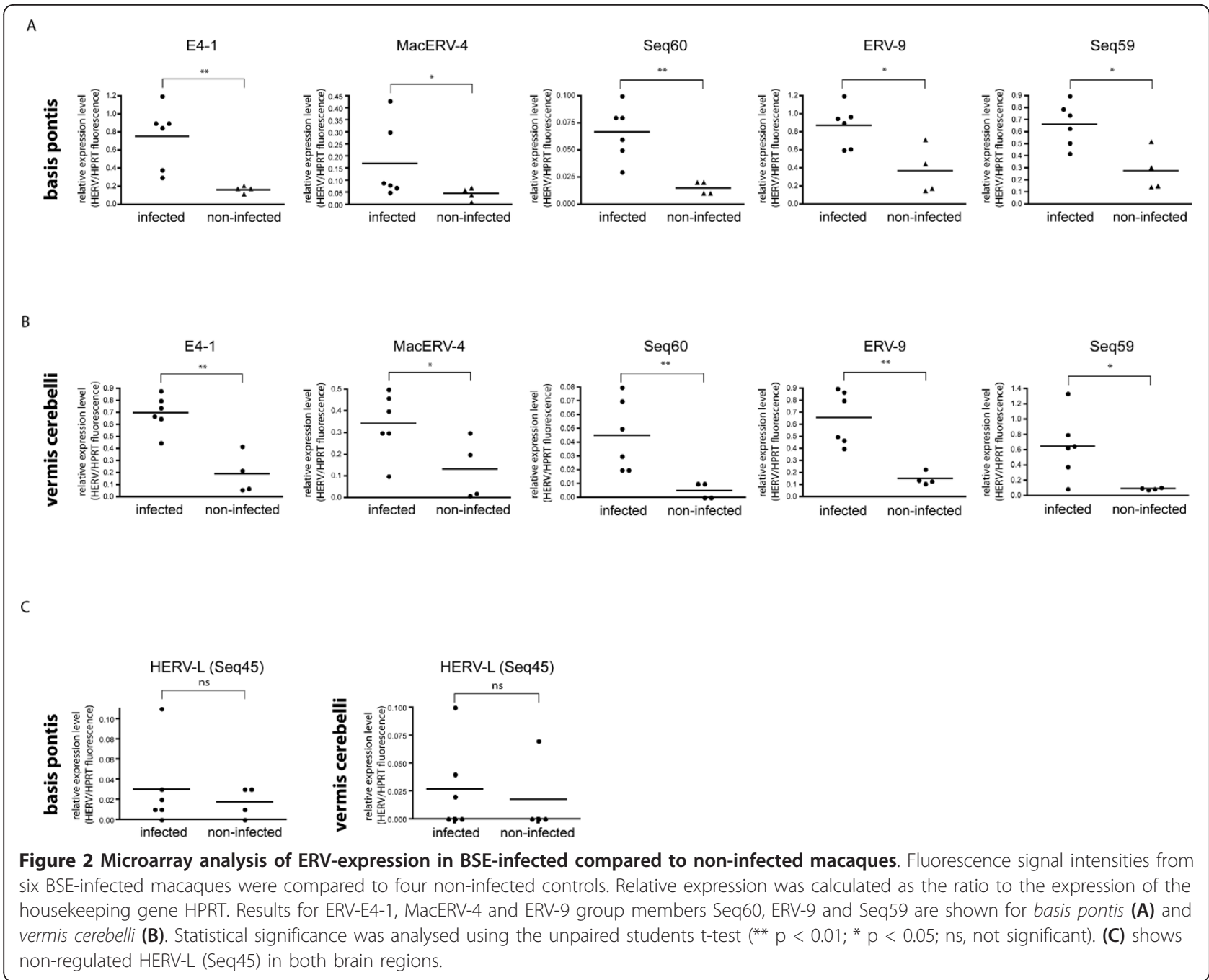

\section{Validation of differentially expressed ERVs in BSE-infected macaques by QPCR}

The retrovirus-specific microarray is a powerful tool for the comprehensive expression analysis and comparison of multiple ERVs in one single experiment. However, the approach cannot differentiate between individual group members of the tested ERVs. Consequently the specificity of hybridization data can be increased by the use of assays designed for the quantification of defined transcripts in a given sample. To confirm the microarray results for HERV-E4-1 and MacERV-4 specific PCR primers were designed. To confirm the specificity of the chosen oligonucleotides the generated PCR products were checked for specific products of the correct size after amplification from cynomolgus macaque-derived cDNA (data not shown) prior to performing the quantitative assays. The same was done for the housekeeping gene chosen, RNA polymerase II (RPII). The primers for HERV-E4-1 have been successfully applied to barbary macaques [40]. Using QPCR we could confirm a significantly elevated HERV-E4-1 expression in the BSEinfected animals as compared to the non-infected controls in the basis pontis (Figure 3A) as well as in the vermis cerebelli (Figure 3B). In addition HERV-K-(HML-3) and the macaque-specific ERV MacERV-4 showed a statistically significant increase in expression upon BSEinfection in both tested brain regions. Since the control animals were not mock infected we cannot rule out that expression of endogenous retroviruses was influenced by the inoculation procedure itself. However, the regions used for our study were spatially separated from the site of inoculation. In addition, our expression analysis was performed approximately 3 years post inoculation which virtually excludes persistent effects triggered by injection of tissue homogenate. In summary, we have identified and validated three ERVs that exhibit an increased expression upon BSE-infection of cynomolgus macaques using two independent techniques, microarray and 


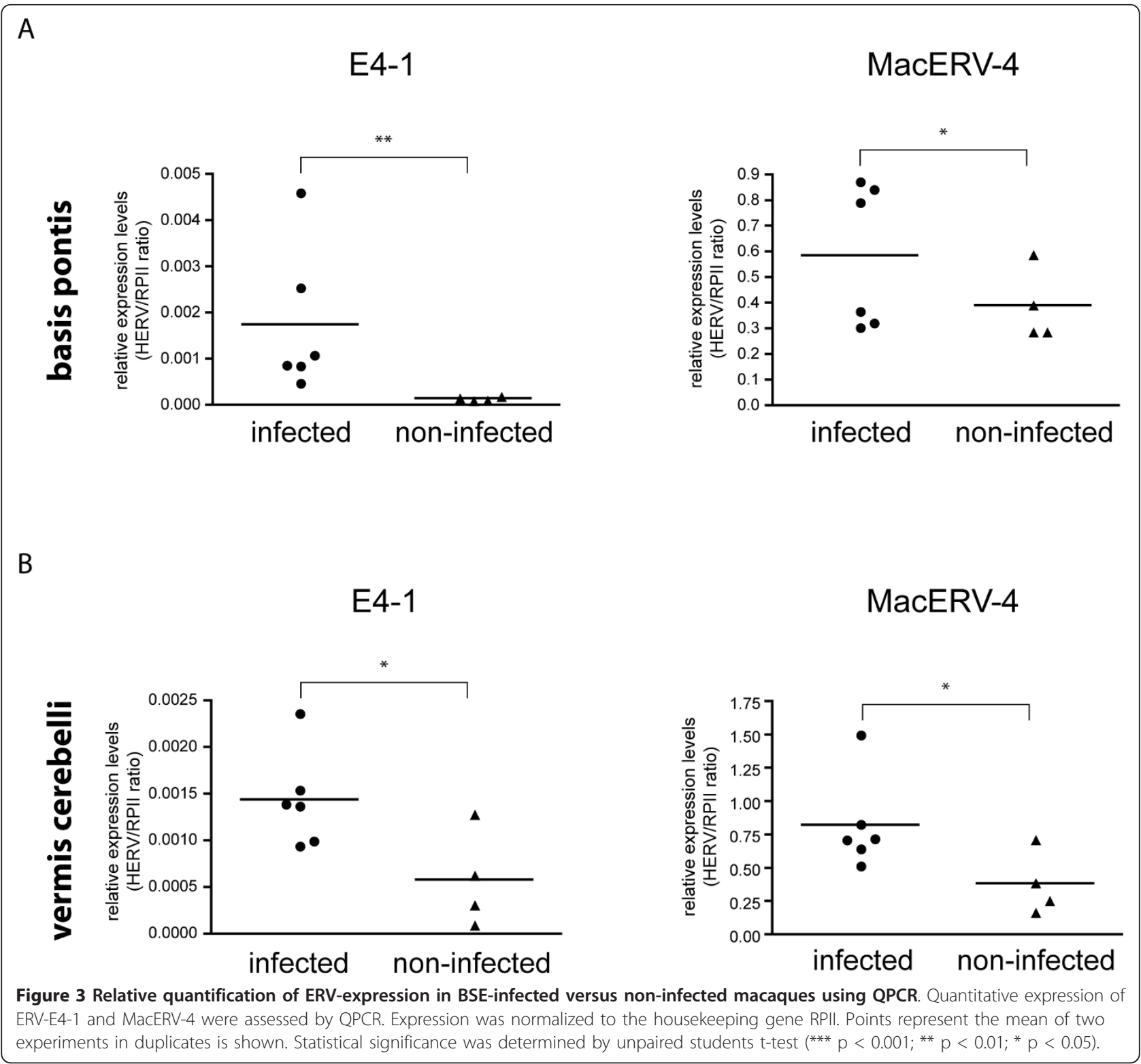

QPCR. We could show that this differential expression exhibited comparable levels in both of the investigated regions, basis pontis and vermis cerebelli.

\section{Comparison of ERV-K-(HML-2) Gag protein levels in the frontal/parietal cortex from BSE-infected and non-infected macaques}

Only a few antibodies against ERVs are commercially available. Even fewer have been tested against non-human primates. Unfortunately, to our knowledge, antibodies directed against polypeptides encoded by the BSE-induced ERVs HERV-E4-1, HERV-K-(HML-3), ERV-9 and MacERV-4 do not exist. We tested a panel of ERV-antibodies (kindly provided by J. Mayer) against
Homo sapiens ERV-K-(HML-2) Gag and Env for crossreactivity with macaque ERV-K-(HML-2) proteins in cortical samples from Macaca fascicularis. Most of the tested antibodies showed low specificity (K1182, polyclonal) or no signal (monoclonal antibodies 6E2-1-4, 3H11 and HERM-1831-5) (data not shown). However, polyclonal antibody K2548 that has been raised against HERVK-(HML-2) Gag [45] exhibited specific bands (Figure 4). Proteins at 70, 80 and $105 \mathrm{kDa}$, respectively, showed an identical migration pattern in simian brain as compared to a human brain sample. Furthermore, the band at 80 $\mathrm{kDa}$ coincides with the $80 \mathrm{kDa}$ full-length Gag protein that is secreted from teratocarcinoma cells (Tera-1) cells [47]. Both, incubation with secondary antibody alone 


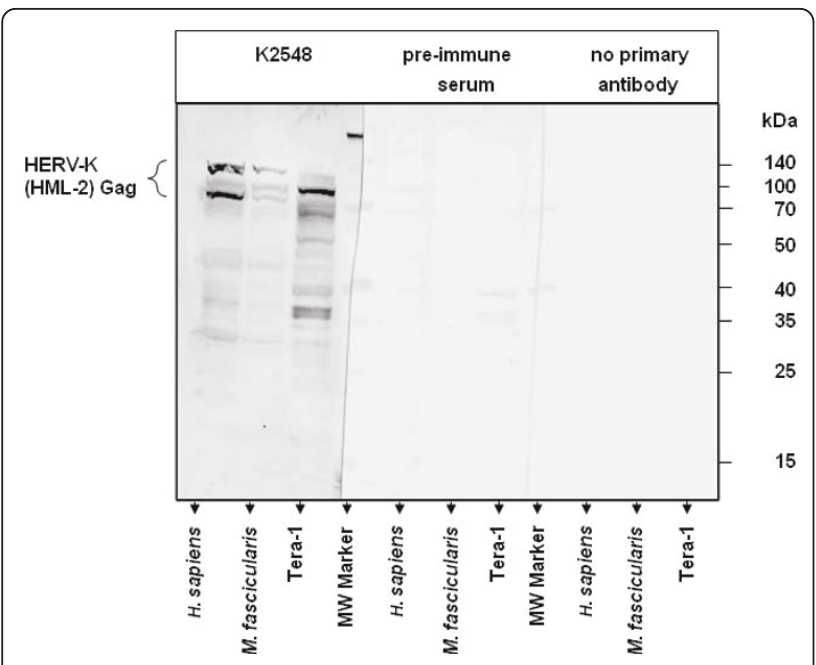

Figure 4 Specific detection of ERV-K-(HML-2) Gag in brain samples derived from macaques and humans. Extracts from the frontal/parietal cortex region of $M$. fascicularis and $H$. sapiens, respectively, and lysates from Tera-1 cells (kindly provided by Dr. J. Mayer) were separated by PAGE and blotted to PVDF membrane. Detection was performed with polyclonal antibody K2548 (left panel), pre-immune serum (middle) or by omission of the primary antibody (right panel), respectively. Predominant HERV-K-(HML-2) Gag immunoreactivity was detected at 70, 80 and $105 \mathrm{kDa}$, but was absent in the pre-immune and secondary antibody controls. Molecular weight marker bands are indicated at the right.

and the respective pre-immune serum did not produce any signal in the respective molecular weight range, confirming that the K2548 antiserum specifically detects macaque ERV-K-(HML-2) Gag. Using this panel of positive and negative controls we concluded that antiserum K2548 could specifically detect macaque ERV-K(HML-2) Gag. It should be mentioned that the probes and antisera used in our study were not developed for the use in cynomolgus macaques. However, macaques and humans are very closely related species on the DNA and on the protein level. In addition, we have demonstrated that the used antiserum detects the same proteins in human and Macaca fascicularis brain samples. This suggests that the cross-reactivity is sufficient to support our conclusions.

We next addressed the question whether macaque ERV-K-(HML-2) Gag protein was differentially expressed in BSE-infected compared to non-infected macaques. Western blot analysis revealed a considerably lower abundance of macaque ERV-K-(HML-2) Gag in the prion-infected animals (Figure 5A). To quantify the difference in the protein levels we performed a densitometric analysis. Normalization to the co-detected beta-actin revealed that macaque ERV-K-(HML-2) Gag protein expression was significantly decreased in BSEinfected macaques as compared to non-infected individuals (Figure 5B). This reduction was confirmed in three independent Western blot experiments and by densitometric analysis of total protein staining using Ponceau S (data not shown).

To analyse whether the downregulation was mirrored at the RNA-level we performed a macaque ERV-K(HML-2) gag specific QPCR on RNA samples derived from the frontal/parietal cortex. A significant downregulation of ERV-K-(HML-2) RNA expression was observed in infected animals, which is consistent with the protein expression data for this brain region (Figure 5C). To our knowledge this is the first report of the regulation of an endogenous virus protein coinciding with prion disease in cynomolgus macaques as a model for CreutzfeldtJacob disease.

\section{Discussion}

Although regarded as a part of the "Junk DNA", ERVs have been shown to play unique functional roles in cellular biology and are implicated in a wide range of diseases like cancer, autoimmune disease, and neurodegeneration [48-51]. We first compared the expression profiles of our old world monkeys (Macaca fascicularis) to existing human profiles $[41,46,52]$. Beside a broad number of similarities, we identified differences in the expression profiles between humans and macaques when comparing Class II betaretroviruses. The HERV-K-(HML-2, -6, and -9) that are strongly expressed in human brain are at the detection limit in macaque brain.

This result is consistent with prior studies of Old World Monkey ERV expression in brain compared to human using a comparable microarray and QPCR [40]. In addition, HERV-K-(HML-3) is more strongly expressed in the basis pontis as compared to the vermis cerebelli of cynomolgus macaque brain. This shows that regional specific differences in ERV expression for class II beta-retroviruses exist in macaques arguing for a functional relevance of these transcripts. We cannot judge whether this finding depicts a general phenomenon. However, the brain region specific expression of ERVs may provide additional insights into molecular mechanisms regulated by ERVs.

Using our non-human primate model we next examined the regulation of endogenous retrovirus expression upon BSE-infection. To date, prion-infected macaques have not been used for analysis of ERV-expression. Differential expression of ERVs upon prion disease have been previously reported in other species, including humans, though brain material has not been examined [28]. Here we provide evidence that ERVs are differentially expressed in an experimentally controlled macaque model of BSE-infection which is highly relevant for human CJD. We have identified the upregulation of ERVs E4-1, MacERV-4, and ERV-9 by ERV-specific microarray. Interestingly, ERV-9 has also been shown to 


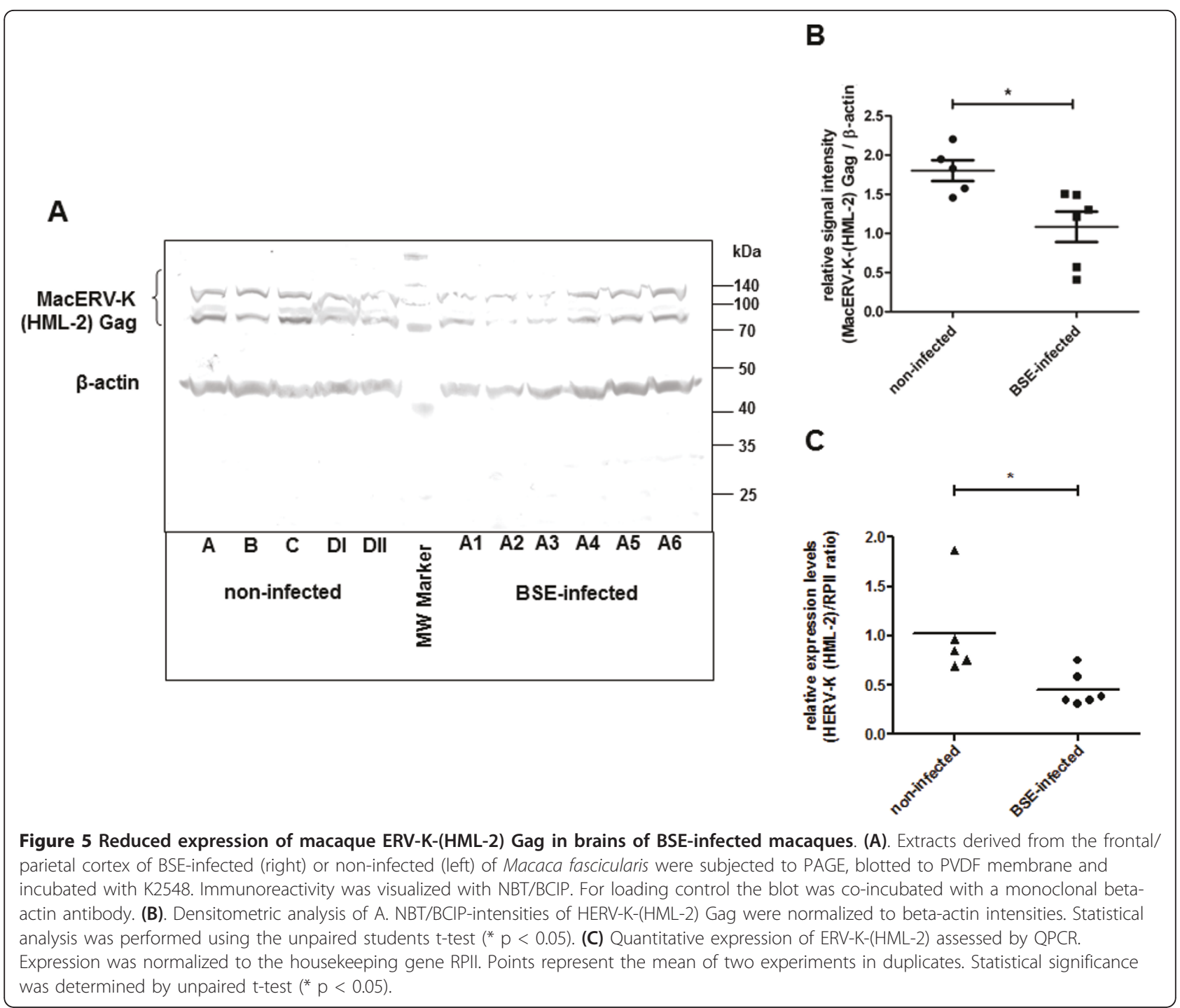

be upregulated in cerebral spinal fluid of SCJD patients [28]. Thus, the macaque-adapted BSE and human SCJD profiles show common ERV-regulation patterns in response to prion infection irrespective of the analysed sample types (brain or cerebrospinal fluid).

We could not judge whether the regulation of ERVs is cause or consequence of prion infection. Although many in vivo studies have been performed, the mechanism connecting prion deposition and neuronal loss has not been determined in detail. The spatial distribution of $\mathrm{PrP}^{\mathrm{Sc}}$ aggregates in different brain regions is not always coincident with neuronal decay in these areas. Thus it appears that neurodegeneration itself could be promoted by biological pathways that are more complex than protein aggregation, possibly by analogous mechanisms as described for neurotropic retroviruses. Indeed, it is known that some retroviruses can induce neurodegeneration in the absence of infectious prions. Different classes of leukemia retroviruses, including CasBrE, 10A1-MuLV, ts1MoMuLV-TB and MoAmphoV induce fatal spongiform encephalomyelopathy [35-37]. Symptoms include tremor, wasting and paraparesis that is caused by neuronal loss, spongiform lesions and astrogliosis, which is intriguingly similar to prion disease pathology [53]. All four retroviruses are classified as gammaretroviruses, the same subgroup as the here described BSE-upregulated MacERV-4, ERV-9 and HERV-E. Thus, changes in ERV expression may induce or exacerbate the pathological consequences of neurodegeneration.

Upregulation of endogenous retroviruses can also potentially induce neurodegeneration. It has been shown that the regulatory LTR-region of HERV-E regulates the expression of the Opitz-syndrome associated mid1 gene [54] that is essential for vermis cerebelli development [55]. It has been proposed that overexpression of mid1 leads to disturbance in microtubule-homeostasis, 
comparable to the tau-aggregation induced neuronal loss in Alzheimer's disease [56,57]. Thus upregulation of HERV-E in BSE-infected macaques may also contribute to neuronal decay similar to the above mechanism. This suggests that induction of retroviral elements may be a consequence of prion infection, and a downstream mechanism of neurodegeneration [36]. This, however, does not imply that endogenous retroviruses can cause prion disease. The appearance of viral particles in prioninfected cells $[58,59]$ has been discussed controversially. Interestingly, recent work has identified $\operatorname{PrP}^{C}$ as an integral component of the Human Immunodeficiency Virus1 (HIV-1) [60]. After cells are infected with HIV-1, the virus replicates, assembles and buds preferentially from the lipid rafts [61] presumably including the GPIanchored $\operatorname{PrP}^{C}$ into the surface of the HIV-1 particles. In addition, HIV-1 Gag and Env colocalize with $\mathrm{PrP}^{\mathrm{C}}$ in infected T-cell lines [62]. Furthermore, expression of HIV-1 gag increases the susceptibility to and sustains the prion infection in cell culture $[26,63]$. Thus spreading of prion infection from one cell to another may not be restricted to exosomal vesicle transfer [64], but may also be triggered by Gag of endogenous origin, such as MacERV-K-(HML-2) Gag. In line with this, murine N2a cells express, produce, and release MuLV (murine-leukaemia virus) particles of endogenous origin, called NeRV (neuroblastoma endogenous retrovirus) [65]. Infection of N2a cells with prions leads to the excretion of exosomes that harbor $\operatorname{PrP}^{\mathrm{C}}$ or infectious $\operatorname{PrP}^{\mathrm{Sc}}$. Intriguingly, antibodies against $\operatorname{PrP}^{\mathrm{C}}$ could label both, exosomes and infectious virions [66]. This suggests that intercellular trafficking of prions could at least partially be mediated by hitchhiking on endogenous retroviral particles. This is in line with earlier observations that PrP can interact with retroviral RNA [67-69] eventually resulting in the formation of active nucleoprotein structures [70] that include Gag. Using specific antibodies we could show that macERV-K-(HML-2) Gag protein is expressed in the brains of cynomolgus macaques. To our knowledge this is the first time that a structural element of an endogenous retrovirus has been detected in the primate central nervous system. The functional role of the protein in neuronal physiology is unknown. Interestingly, among the many endogenous retroviral subfamilies, HERV-K-(HML-2) can produce viral particles [71-74]. It remains unclear whether macERV (HML-2) Gag can be excreted by neurons. However, active release of HERV-K-(HML-2) Gag containing retrovirus-like particles have been described in Tera-1 [47] cells and were also found in blood plasma of patients $[75,76]$. Taken together, the detection of macERV-K-(HML-2) protein in the frontal/parietal cortex indicates that it may have a physiological role in the brain. Furthermore, finding that HERV-K-(HML-2) Gag protein and RNA is downregulated in BSE-infected macaques suggests that this role may be connected to neuronal survival. Further studies will be necessary to determine the mechanism and function of HERV-K HML-2 Gag downregulation.

\section{Conclusions}

We could show that prion disease in a non-human primate model is associated with the alteration of ERVexpression at the transcriptional and the protein level. Upregulated expression of ERVs in macaques demonstrated partial overlap to those found in CSF from human CJD patients, underlining that our model is especially suitable to mimic the pathology of human TSEs. This is consistent with the hypothesis that endogenous retroviruses may provide a missing link between protein aggregation and neuronal loss in prion diseases. It is reasonable to speculate that the putatively secreted HERV-K (HML-2) Gag may contribute to the survival of neuronal cells.

Based on our findings, further research using specialized models may help to elucidate the association between prions and endogenous retroviruses and its putative role in neurodegeneration.

\section{Additional material}

Additional file 1: Figures S1, S2, and S3.

\section{Acknowledgements}

The authors thank Jens Mayer and Marlies Sauter for providing HERV-K antibodies, Tera-1 cell lysates, and helpful advice on the cross species Western blot experiments. The authors thank Christine Leib-Mösch for her support and helpful discussions. We also thank Walter Schulz-Schaeffer for brain sample preparation and Sabine Borchert and Mareike Curth for excellent assistance in performing the Western blot and QPCR analysis. This work was supported by funds of the European Community (BSE transmission to primates, QLK1-CT-2002-01096).

\section{Author details}

'Leibniz-Institute for Zoo and Wildlife Research, Alfred-Kowalke Str. 17, D10315 Berlin, Germany. ${ }^{2}$ Institute of Virology, Helmholtz Zentrum München, Ingolstaedter Landstr. 1, D-85764 Neuherberg, Germany. ${ }^{3}$ German Primate Center, Leibniz-Institute for Primate Research, Unit of Infection Models, D37077 Göttingen, Germany. ${ }^{4}$ III Medizinische Universitätsklinik, Medizinische Fakultät Mannheim der Universität Heidelberg, D-68305 Mannheim, Germany. ${ }^{5}$ Institute of Toxicology, Helmholtz Zentrum München, Ingolstaedter Landstr. 1, D-85764 Neuherberg, Germany. ${ }^{6}$ Molecular and Cell Physiology, Hannover Medical School, Carl-Neuberg-Str. 1, D-30625 Hannover, Germany.

\section{Authors' contributions}

ADG and MOZ designed the study. MV and WS established the microarray and carried out the experiments. MV carried out the QPCR experiments. ACS carried out the Western Blot analysis. JM carried out the RNA sample preparations from simian tissues. MV and ACS were responsible for the data analysis. ADG, JM and MOZ wrote the manuscript with contributions from all authors. All authors read and approved the final manuscript.

\section{Competing interests}

The authors declare that they have no competing interests. 
Received: 24 February 2011 Accepted: 23 June 2011

Published: 23 June 2011

\section{References}

1. Chesebro B: Introduction to the transmissible spongiform encephalopathies or prion diseases. British medical bulletin 2003, 66:1-20

2. Nunziante M, Gilch S, Schatzl HM: Prion diseases: from molecular biology to intervention strategies. Chembiochem 2003, 4:1268-1284.

3. Aguzzi A, Polymenidou M: Mammalian prion biology: one century of evolving concepts. Cell 2004, 116:313-327.

4. Priola SA, Vorberg I: Molecular aspects of disease pathogenesis in the transmissible spongiform encephalopathies. Methods in molecular biology (Clifton, NJ 2004, 268:517-540.

5. Prusiner SB: Molecular biology of prion diseases. Science (New York, NY 1991, 252:1515-1522

6. Pan KM, Baldwin M, Nguyen J, Gasset M, Serban A, Groth D, Mehlhorn I, Huang Z, Fletterick RJ, Cohen FE, et al: Conversion of alpha-helices into beta-sheets features in the formation of the scrapie prion proteins. Proceedings of the National Academy of Sciences of the USA 1993, 90:10962-10966.

7. Caughey B, Baron GS: Prions and their partners in crime. Nature 2006, 443:803-810

8. Kaneko K, Zulianello L, Scott M, Cooper CM, Wallace AC, James TL, Cohen FE, Prusiner SB: Evidence for protein $\times$ binding to a discontinuous epitope on the cellular prion protein during scrapie prion propagation. Proceedings of the National Academy of Sciences of the USA 1997, 94:10069-10074

9. Hachiya NS, Imagawa M, Kaneko K: The possible role of protein X, a putative auxiliary factor in pathological prion replication, in regulating a physiological endoproteolytic cleavage of cellular prion protein. Medical hypotheses 2007, 68:670-673.

10. Lee Cl, Yang Q, Perrier V, Baskakov IV: The dominant-negative effect of the Q218K variant of the prion protein does not require protein X. Protein SCi 2007, 16:2166-2173.

11. Doh-ura K, Perryman S, Race R, Chesebro B: Identification of differentially expressed genes in scrapie-infected mouse neuroblastoma cells. Microbial pathogenesis 1995, 18:1-9.

12. Riemer C, Queck I, Simon D, Kurth R, Baier M: Identification of upregulated genes in scrapie-infected brain tissue. Journal of virology 2000, 74:10245-10248

13. Riemer C, Neidhold S, Burwinkel M, Schwarz A, Schultz J, Kratzschmar J, Monning U, Baier M: Gene expression profiling of scrapie-infected brain tissue. Biochemical and biophysical research communications 2004, 323:556-564

14. Baker CA, Manuelidis $L$ : Unique inflammatory RNA profiles of microglia in Creutzfeldt-Jakob disease. Proceedings of the National Academy of Sciences of the USA 2003, 100:675-679.

15. Xiang W, Windl O, Wunsch G, Dugas M, Kohlmann A, Dierkes N, Westner IM, Kretzschmar HA: Identification of differentially expressed genes in scrapie-infected mouse brains by using global gene expression technology. Journal of virology 2004, 78:11051-11060

16. Brown AR, Rebus S, McKimmie CS, Robertson K, Williams A, Fazakerley JK: Gene expression profiling of the preclinical scrapie-infected hippocampus. Biochemical and biophysical research communications 2005, 334:86-95.

17. Greenwood AD, Horsch M, Stengel A, Vorberg I, Lutzny G, Maas E, Schadler S, Erfle V, Beckers J, Schatzl H, Leib-Mosch C: Cell line dependent RNA expression profiles of prion-infected mouse neuronal cells. Journal of molecular biology 2005, 349:487-500.

18. Sawiris GP, Becker KG, Elliott EJ, Moulden R, Rohwer RG: Molecular analysis of bovine spongiform encephalopathy infection by CDNA arrays. The Journal of general virology 2007, 88:1356-1362.

19. Martinez T, Pascual A: Identification of genes differentially expressed in SH-SY5Y neuroblastoma cells exposed to the prion peptide 106-126. The European journal of neuroscience 2007, 26:51-59.

20. Bach C, Gilch S, Rost R, Greenwood AD, Horsch M, Hajj GN, Brodesser S, Facius A, Schadler S, Sandhoff K, et al: Prion-induced activation of cholesterogenic gene expression by Srebp2 in neuronal cells. The Journal of biological chemistry 2009, 284:31260-31269.
21. Benetti F, Gasperini L, Zampieri M, Legname G: Gene expression profiling to identify druggable targets in prion diseases. Expert Opinion on Drug Discovery 2010, 5:177-202

22. Montag J, Hitt R, Opitz L, Schulz-Schaeffer WJ, Hunsmann G, Motzkus D: Upregulation of miRNA hsa-miR-342-3p in experimental and idiopathic prion disease. Molecular neurodegeneration 2009, 4:36.

23. Julius C, Hutter G, Wagner U, Seeger H, Kana V, Kranich J, Klohn PC, Weissmann C, Miele G, Aguzzi A: Transcriptional stability of cultured cells upon prion infection. Journal of molecular biology 2008, 375:1222-1233.

24. Carp Rl, Meeker HC, Caruso V, Sersen E: Scrapie strain-specific interactions with endogenous murine leukaemia virus. The Journal of general virology 1999, 80(Pt 1):5-10

25. Stengel A, Bach C, Vorberg I, Frank O, Gilch S, Lutzny G, Seifarth W, Erfle V, Maas $E$, Schatzl $H$, et al: Prion infection influences murine endogenous retrovirus expression in neuronal cells. Biochemical and biophysical research communications 2006, 343:825-831.

26. Leblanc P, Alais S, Porto-Carreiro I, Lehmann S, Grassi J, Raposo G, Darlix JL: Retrovirus infection strongly enhances scrapie infectivity release in cell culture. The EMBO journal 2006, 25:2674-2685.

27. Lee KH, Jeong BH, Jin JK, Meeker HC, Kim JI, Carp RI, Kim YS: Scrapie infection activates the replication of ecotropic, xenotropic, and polytropic murine leukemia virus (MuLV) in brains and spinal cords of senescence-accelerated mice: implication of MuLV in progression of scrapie pathogenesis. Biochemical and biophysical research communications 2006, 349:122-130

28. Jeong BH, Lee YJ, Carp RI, Kim YS: The prevalence of human endogenous retroviruses in cerebrospinal fluids from patients with sporadic Creutzfeldt-Jakob disease. J Clin Virol 2010, 47:136-142.

29. Denner J: Endogenous Retroviruses. In Retroviruses: Molecular Biology, Genomics and Pathogenesis. Edited by: Kurth R, Bannert N. Hethersett, Norwich, UK: Caister Academic Press; 2010:35-69.

30. Blaise S, de Parseval N, Benit L, Heidmann T: Genomewide screening for fusogenic human endogenous retrovirus envelopes identifies syncytin 2 , a gene conserved on primate evolution. Proceedings of the National Academy of Sciences of the USA 2003, 100:13013-13018.

31. Dupressoir A, Marceau G, Vernochet C, Benit L, Kanellopoulos C, Sapin V Heidmann T: Syncytin-A and syncytin-B, two fusogenic placenta-specific murine envelope genes of retroviral origin conserved in Muridae. Proceedings of the National Academy of Sciences of the USA 2005, 102:725-730.

32. Peng X, Pan J, Gong R, Liu Y, Kang S, Feng H, Qiu G, Guo D, Tien P, Xiao G: Functional characterization of syncytin- $A$, a newly murine endogenous virus envelope protein. Implication for its fusion mechanism. The Journal of biological chemistry 2007, 282:381-389.

33. Heidmann O, Vernochet C, Dupressoir A, Heidmann T: Identification of an endogenous retroviral envelope gene with fusogenic activity and placenta-specific expression in the rabbit: a new "syncytin" in a third order of mammals. Retrovirology 2009, 6:107.

34. Lotscher M, Recher M, Lang KS, Navarini A, Hunziker L, Santimaria R, Glatzel M, Schwarz P, Boni J, Zinkernagel RM: Induced prion protein controls immune-activated retroviruses in the mouse spleen. PloS one 2007, 2:e1158.

35. Szurek PF, Yuen PH, Jerzy R, Wong PK: Identification of point mutations in the envelope gene of Moloney murine leukemia virus TB temperaturesensitive paralytogenic mutant ts1: molecular determinants for neurovirulence. Journal of virology 1988, 62:357-360.

36. Jolicoeur P, Masse G, Kay DG: The prion protein gene is dispensable for the development of spongiform myeloencephalopathy induced by the neurovirulent Cas-Br-E murine leukemia virus. Journal of virology 1996, 70:9031-9034.

37. Munk C, Prassolov V, Rodenburg M, Kalinin V, Lohler J, Stocking C: 10A1MuLV but not the related amphotropic 4070A MuLV is highly neurovirulent: importance of sequences upstream of the structural Gag coding region. Virology 2003, 313:44-55.

38. Lasmezas Cl, Comoy E, Hawkins S, Herzog C, Mouthon F, Konold T, Auvre F, Correia E, Lescoutra-Etchegaray N, Sales N, et al: Risk of oral infection with bovine spongiform encephalopathy agent in primates. Lancet 2005, 365:781-783.

39. Greenwood AD, Stengel A, Erfle V, Seifarth W, Leib-Mosch C: The distribution of pol containing human endogenous retroviruses in nonhuman primates. Virology 2005, 334:203-213. 
40. Stengel A, Roos C, Hunsmann G, Seifarth W, Leib-Mosch C, Greenwood AD: Expression profiles of endogenous retroviruses in Old World monkeys. Journal of virology 2006, 80:4415-4421.

41. Seifarth W, Frank O, Zeilfelder U, Spiess B, Greenwood AD, Hehlmann R, Leib-Mosch C: Comprehensive analysis of human endogenous retrovirus transcriptional activity in human tissues with a retrovirus-specific microarray. Journal of virology 2005, 79:341-352.

42. Seifarth W, Spiess B, Zeilfelder U, Speth C, Hehlmann R, Leib-Mosch C: Assessment of retroviral activity using a universal retrovirus chip. Journal of virological methods 2003, 112:79-91.

43. Radonic A, Thulke S, Mackay IM, Landt O, Siegert W, Nitsche A: Guideline to reference gene selection for quantitative real-time PCR. Biochemical and biophysical research communications 2004, 313:856-862.

44. Pfaffl MW: A new mathematical model for relative quantification in realtime RT-PCR. Nucleic acids research 2001, 29:e45.

45. Sauter M, Schommer S, Kremmer E, Remberger K, Dolken G, Lemm I, Buck M, Best B, Neumann-Haefelin D, Mueller-Lantzsch N: Human endogenous retrovirus K10: expression of Gag protein and detection of antibodies in patients with seminomas. Journal of virology 1995, 69:414-421.

46. Frank O, Giehl M, Zheng C, Hehlmann R, Leib-Mosch C, Seifarth W: Human endogenous retrovirus expression profiles in samples from brains of patients with schizophrenia and bipolar disorders. Journal of virology 2005, 79:10890-10901.

47. Gotzinger N, Sauter M, Roemer K, Mueller-Lantzsch N: Regulation of human endogenous retrovirus-K Gag expression in teratocarcinoma cell lines and human tumours. The Journal of general virology 1996, 77(Pt 12):2983-2990.

48. Balada E, Vilardell-Tarres M, Ordi-Ros J: Implication of human endogenous retroviruses in the development of autoimmune diseases. International reviews of immunology 2010, 29:351-370.

49. Christensen T: HERVs in neuropathogenesis. J Neuroimmune Pharmacol 2010, 5:326-335.

50. Kurth R, Bannert N: Beneficial and detrimental effects of human endogenous retroviruses. International journal of cancer 2010, 126:306-314.

51. Ruprecht K, Mayer J, Sauter M, Roemer K, Mueller-Lantzsch N: Endogenous retroviruses and cancer. Cell Mol Life Sci 2008, 65:3366-3382.

52. Forsman A, Yun Z, Hu L, Uzhameckis D, Jern P, Blomberg J: Development of broadly targeted human endogenous gammaretroviral pol-based real time PCRs Quantitation of RNA expression in human tissues. Journal of virological methods 2005, 129:16-30.

53. Clase AC, Dimcheff DE, Favara C, Dorward D, McAtee FJ, Parrie LE, Ron D, Portis $\lrcorner$ : Oligodendrocytes are a major target of the toxicity of spongiogenic murine retroviruses. The American journal of pathology 2006, 169:1026-1038.

54. Landry JR, Rouhi A, Medstrand P, Mager DL: The Opitz syndrome gene Mid1 is transcribed from a human endogenous retroviral promoter. Molecular biology and evolution 2002, 19:1934-1942.

55. Lancioni A, Pizzo M, Fontanella B, Ferrentino R, Napolitano LM, De Leonibus E, Meroni G: Lack of Mid1, the mouse ortholog of the Opitz syndrome gene, causes abnormal development of the anterior cerebellar vermis. J Neurosci 2010, 30:2880-2887.

56. Cainarca S, Messali S, Ballabio A, Meroni G: Functional characterization of the Opitz syndrome gene product (midin): evidence for homodimerization and association with microtubules throughout the cell cycle. Human molecular genetics 1999, 8:1387-1396.

57. Schweiger S, Foerster J, Lehmann T, Suckow V, Muller YA, Walter G, Davies T, Porter $H$, van Bokhoven $H$, Lunt PW, et al: The Opitz syndrome gene product, MID1, associates with microtubules. Proceedings of the National Academy of Sciences of the USA 1999, 96:2794-2799.

58. Manuelidis L, Yu ZX, Barquero N, Mullins B: Cells infected with scrapie and Creutzfeldt-Jakob disease agents produce intracellular $25-\mathrm{nm}$ virus-like particles. Proceedings of the National Academy of Sciences of the USA 2007, 104:1965-1970.

59. Manuelidis L: A $25 \mathrm{~nm}$ virion is the likely cause of transmissible spongiform encephalopathies. Journal of cellular biochemistry 2007, 100:897-915.

60. Alais S, Georges G, Berlioz-Torrent C, Leblanc P: Focus on the antiviral properties of the cellular prion protein PrPC/CD230. Prion 2010, 4:187.

61. Campbell SM, Crowe SM, Mak J: Lipid rafts and HIV-1: from viral entry to assembly of progeny virions. J Clin Virol 2001, 22:217-227.
62. Leblanc P, Baas D, Darlix JL: Analysis of the interactions between HIV-1 and the cellular prion protein in a human cell line. Journal of molecular biology 2004, 337:1035-1051.

63. Bian J, Napier D, Khaychuck V, Angers R, Graham C, Telling G: Cell-based quantification of chronic wasting disease prions. Journal of virology 2010, 84:8322-8326.

64. Fevrier B, Vilette D, Archer F, Loew D, Faigle W, Vidal M, Laude H, Raposo G Cells release prions in association with exosomes. Proceedings of the National Academy of Sciences of the USA 2004, 101:9683-9688.

65. Pothlichet J, Heidmann T, Mangeney M: A recombinant endogenous retrovirus amplified in a mouse neuroblastoma is involved in tumor growth in vivo. International journal of cancer 2006, 119:815-822.

66. Alais S, Simoes S, Baas D, Lehmann S, Raposo G, Darlix JL, Leblanc P: Mouse neuroblastoma cells release prion infectivity associated with exosomal vesicles. Biology of the cell/under the auspices of the European Cell Biology Organization 2008, 100:603-615.

67. Nandi PK, Nicole JC: Nucleic acid and prion protein interaction produces spherical amyloids which can function in vivo as coats of spongiform encephalopathy agent. Journal of molecular biology 2004, 344:827-837.

68. Deleault NR, Lucassen RW, Supattapone S: RNA molecules stimulate prion protein conversion. Nature 2003, 425:717-720

69. Gabus C, Auxilien S, Pechoux C, Dormont D, Swietnicki W, Morillas M, Surewicz W, Nandi P, Darlix JL: The prion protein has DNA strand transfer properties similar to retroviral nucleocapsid protein. Journal of molecular biology 2001, 307:1011-1021.

70. Gabus C, Derrington E, Leblanc P, Chnaiderman J, Dormont D, Swietnicki W, Morillas M, Surewicz WK, Marc D, Nandi P, Darlix JL: The prion protein has RNA binding and chaperoning properties characteristic of nucleocapsid protein NCP7 of HIV-1. The Journal of biological chemistry 2001, 276:19301-19309.

71. Bannert N, Kurth R: Retroelements and the human genome: new perspectives on an old relation. Proceedings of the National Academy of Sciences of the USA 2004, 101(Suppl 2):14572-14579.

72. Simpson GR, Patience C, Lower R, Tonjes RR, Moore HD, Weiss RA, Boyd MT Endogenous D-type (HERV-K) related sequences are packaged into retroviral particles in the placenta and possess open reading frames for reverse transcriptase. Virology 1996, 222:451-456.

73. Bieda K, Hoffmann A, Boller K: Phenotypic heterogeneity of human endogenous retrovirus particles produced by teratocarcinoma cell lines. The Journal of general virology 2001, 82:591-596.

74. Boller K, Konig H, Sauter M, Mueller-Lantzsch N, Lower R, Lower J, Kurth R: Evidence that HERV-K is the endogenous retrovirus sequence that codes for the human teratocarcinoma-derived retrovirus HTDV. Virology 1993, 196:349-353.

75. Contreras-Galindo R, Kaplan MH, Markovitz DM, Lorenzo E, Yamamura Y: Detection of HERV-K(HML-2) viral RNA in plasma of HIV type 1-infected individuals. AIDS research and human retroviruses 2006, 22:979-984.

76. Contreras-Galindo R, Kaplan MH, Leissner P, Verjat T, Ferlenghi I, Bagnoli F, Giusti F, Dosik MH, Hayes DF, Gitlin SD, Markovitz DM: Human endogenous retrovirus $\mathrm{K}(\mathrm{HML}-2)$ elements in the plasma of people with lymphoma and breast cancer. Journal of virology 2008, 82:9329-9336.

doi:10.1186/1750-1326-6-44

Cite this article as: Greenwood et al: Bovine spongiform

encephalopathy infection alters endogenous retrovirus expression in distinct brain regions of cynomolgus macaques (Macaca fascicularis). Molecular Neurodegeneration 2011 6:44. 